ASM Sc. J., 13, 2020

https://doi.org/10.32802/asmscj.2020.sm26(1.22)

\title{
The Energy of Cayley Graphs for Symmetric Groups of Order 24
}

\author{
Amira Fadina Ahmad Fadzil ${ }^{*}$, Nor Haniza Sarmin ${ }^{2}$ and Ahmad Erfanian ${ }^{3}$ \\ 1,2 Department of Mathematical Sciences, Faculty of Science, Universiti Teknologi Malaysia, \\ 81310 UTM Johor Bahru, Johor, Malaysia. \\ ${ }^{3}$ Department of Pure Mathematics, Faculty of Mathematical Science, and Center of Excellence in \\ Analysis on Algebraic Structures, Ferdowsi University of Mashhad, Mashhad, Iran.
}

\begin{abstract}
A Cayley graph of a finite group $G$ with respect to a subset $S$ of $G$ is a graph where the vertices of the graph are the elements of the group and two distinct vertices $x$ and $y$ are adjacent to each other if $x y^{-1}$ is in the subset $S$. The subset of the Cayley graph is inverse closed and does not include the identity of the group. For a simple finite graph, the energy of a graph can be determined by summing up the positive values of the eigenvalues of the adjacency matrix of the graph. In this paper, the graph being studied is the Cayley graph of symmetric group of order 24 where $S$ is the subset of $S_{4}$ of valency up to two. From the Cayley graphs, the eigenvalues are calculated by constructing the adjacency matrix of the graphs and by using some properties of special graphs. Finally, the energy of the respected Cayley graphs is computed and presented.
\end{abstract}

Keywords: energy of graph; cayley graph; symmetric groups

\section{INTRODUCTION}

The study on Cayley graphs was initiated by Arthur Cayley in 1878 to explain the idea of abstract groups described by a set of generators (Ghorbani \& Nowroozi-Larki, 2018). The theory has been advanced into a significant branch in algebraic graph theory. There are many problems regarding Cayley graphs that have been studied by many graph theorists and group theorists.

In 1988, Babai and Seress have studied on the diameter of the Cayley graphs of symmetric groups and the alternating groups. Not long after, in 1993, Lakshmivarahan et. al., have analysed the symmetries in the interconnection networks of a variety of Cayley graphs of permutation groups. The types of symmetry analysed consist of vertex and edge transitivity, distance regularity and distance transitivity

Meanwhile in 2000, Friedman has shown in his study that among all sets of $n-1$ transpositions which generate the symmetric groups, the Cayley graph associated to set $S=$ $\{(1, n),(2, n), \ldots,(n-1, n)\}$ as the highest eigenvalues.
Konstantinova in 2008, has surveyed the historical changes of some problems on Cayley graphs such as the Hamiltonicity and diameter problems. The author also included various uses of Cayley graphs in solving combinatorial, graph theoretical and applied problems. Furthermore in 2012, Adiga and Ariamanesh have determined the number of undirected Cayley graphs of symmetric group and alternating groups up to isomorphism.

In addition, the study on the energy of general simple graphs was first defined by Gutman in 1978 inspired from the Huckel Molecular Orbital (HMO) theory proposed in 1930s. The theory has been used by chemists in approximating the energies related with $\pi$-electron orbitals in conjugated hydrocarbon. Besides, its chemical applications, there are a few applications in other field of science such as in graph entropies (Dehmer et. al., 2015), modelling of properties of proteins (Wu et. al., 2015) and also was used in the search for the genetic causes of Alzheimer disease (Daianu et al., 2015).

Therefore, this paper aimed to present the energy of the Cayley graphs associated to symmetric group of order 24 with

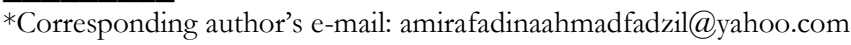


respect to the subsets $S$ of valency one and two. The methodology consists of listing the subsets $S$, constructing the Cayley graphs with respect to the subsets $S$, finding their isomorphism, building the adjacency matrix for the graphs, calculating the eigenvalues of the adjacency matrix of the graphs and computing the energy of the Cayley graphs.

This paper is structured as follows: in Section 1, the early studies for the topics are introduced while in Section 2, the preliminaries results that are being used for this study are presented. In Section 3, the main results are discussed in two propositions and theorems and finally, Section 4 gives the conclusion to the main results.

\section{PRELIMINARIES}

The followings are some basic concepts and properties that are used in this study.

Definition 1 (Robinson, 1996) Symmetric Group

If $X$ is a nonempty set, a bijection $\pi: X \rightarrow X$ is called a permutation of $X$. The set of all permutations of $X$ is a group with respect to functional composition called the symmetric groups on $X, \operatorname{Sym} X$. When $X=\{1,2, \ldots, n\}$, it is customary to write $S_{n}$ for Sym $X$ and to call this the symmetric groups of degree $n$. This study focused on the symmetric group of order 24 denoted as $S_{4}$. The elements of $S_{4}=$ $\{(1),(12),(13),(14),(23),(24),(34),(123)$,

(132), (124), (142), (134), (143), (234), (243),

(1234), (1432), (1243), (1342), (1324), (1423),

(12)(34), (13)(24), (14)(23)\}. This study also focused on the subsets of $S_{4}$ of valency 1 and 2 where the valency of the subsets is the degree of the subsets.

Definition 2 (Beineke \& Wilson, 2004) Cayley Graph of a Group

Let $G$ be a finite group with identity 1 . Let $S$ be a subset of $G$ satisfying $1 \notin S$ and $S=S^{-1}$; that is, $s \in S$ if and only if $s^{-1} \in$ $S$. The Cayley graph $\operatorname{Cay}(G ; S)$ on $G$ with connection set $S$ is defined as follows:

(i) the vertices are the elements of $G$

(ii) there is an edge joining $g$ and $h$ if and only if $h=s g$ for some $s \in S$.

The set of all Cayley graphs on $G$ is denoted by $\operatorname{Cay}(G ; S)$ where $S$ is the subset of $G$ with a certain valency which is the order of $S$.
Definition 3 (Beineke \& Wilson, 2004) Complete Graph A complete graph $K_{n}$ has $n$ vertices, each of which is adjacent to all of the others.

Definition 4 (Pemmaraju \& Skiena, 2003) Cycle Graph

A cycle graph $C_{n}$, sometimes simply known as an $n$-cycle, is a graph on $n$ vertices containing a single cycle through all the vertices.

Proposition 1 (Brouwer \& Haemers, 2011) Spectrum of Complete Graph

Let be the complete graph $K_{n}$ on $n$ vertices. Its spectrum is $\left\{(n-1)^{1},(-1)^{n-1}\right\}$.

Proposition 2 (Brouwer \& Haemers, 2011) Spectrum of Cycle Graph

Consider the undirected $n$-cycle graph $C_{n}$. The spectrum of $C_{n}$ consists of the numbers $\{2 \cos (2 \pi j / n) ; j=\{0,1, \ldots, n-1\}$.

\section{Definition 5 (Bapat, 2010) Adjacency Matrix}

Let $\Gamma$ be a graph with $V(\Gamma)=\{1, \ldots, n\}$ and $E(\Gamma)=\left\{e_{1}, \ldots, e_{m}\right\}$. The adjacency matrix of $\Gamma$ denoted by $A(\Gamma)$ is the $n \times n$ matrix defined as follows. The rows and the columns of $A(\Gamma)$ are indexed by $V(\Gamma)$. If $i \neq j$ then the $(i, j)$-entry of $A(\Gamma)$ is 0 for vertices $i$ and $j$ nonadjacent, and the $(i, j)$-entry is 1 for $i$ and $j$ adjacent. The $(i, i)-\operatorname{entry}$ of $A(\Gamma)$ is 0 for $i=1, \ldots, n$. $A(\Gamma)$ is often simply denoted by $A$.

Definition 6 (Bapat, 2010) Energy of Graph

For any graph $\Gamma$, the energy of the graph is defined as $\varepsilon(\Gamma)=$ $\sum_{i=1}^{n}\left|\lambda_{i}\right|$ where $\lambda_{1}, \ldots, \lambda_{n}$ are the eigenvalues of the adjacency matrix of $\Gamma$.

\section{MAIN RESULTS}

In this section, our main results are specified in terms of propositions and theorems. The propositions present the generalization of the Cayley graphs of $S_{4}$ with respect to the subsets $S$ of valency one and two. Then, the results on the energy of the Cayley graphs found are presented in two theorems. The findings in the propositions are used in proving the theorems following them.

\section{Proposition 3}

Let $S_{4}$ be the symmetric group of order 24 and $S^{(1)}$ be the subset of $S_{4}$ of valency one, $\left|S^{(1)}\right|=1$. Then, the Cayley graph 
of $S_{4}$ with respect to the subset $S^{(1)}$, denoted as $\operatorname{Cay}\left(S_{4}, S^{(1)}\right)$ is $\bigcup_{i=1}^{12} K_{2}$.

\section{Proof}

Suppose $S_{4}$ is the symmetric group of order 24 and $\operatorname{Cay}\left(S_{4}, S^{(1)}\right)$ is the Cayley graph of $S_{4}$ with respect to the subset $S^{(1)}$ of valency one. The vertex set of the Cayley graph, $V\left(\operatorname{Cay}\left(S_{4}, S^{(1)}\right)\right)=\{(1),(12),(13),(14),(23)$,

(24), (34), (123), (132), (124), (142), (134), (143),

(234), (243), (1234), (1432), (1243), (1342),

(1324), (1423), (12)(34), (13)(24), (14)(23)\}.

Then, by the definition of Cayley graph, let $S^{(1)}=\{(12)(34)\}$, the edge set of the Cayley graph, $\operatorname{Edge}\left(\operatorname{Cay}\left(S_{-} 4, S^{(1)}\right)\right)=$ $\{\{(1),(12)(34)\}$,

$\{(12),(34)\},\{(13),(1432)\},\{(14),(1342)\}$,

$\{(23),(1243)\},\{(24),(1234)\},\{(123),(243)\}$,

$\{(132),(143)\},\{(124),(234)\},\{(142),(134)\}$,

$\{(1324),(1423)\},\{(13)(24),(14)(23)\}\}$.

Thus, $\operatorname{Cay}\left(S_{4}, S^{(1)}\right)$ is the union of 12 complete graphs of two vertices, $\mathrm{U}_{i=1}^{12} K_{2}$.

\section{Proposition 4}

Let $S_{4}$ be the symmetric group of order 24 and $S^{(2)}$ be the subset of $S_{4}$ of valency two, $\left|S^{(2)}\right|=2$. Then, the Cayley graph of $S_{4}$ with respect to the subset $S^{(2)}$, denoted as $\operatorname{Cay}\left(S_{4}, S^{(2)}\right)$ is given in the following:

$$
\operatorname{Cay}\left(S_{4}, S^{(2)}\right)= \begin{cases}\bigcup_{i=1}^{4} C_{6} & ; S=\{(i j),(k l)\}, \\ \bigcup_{i=1}^{3} C_{8} & ; S=\{(i j),(k l)(m n)\}, \\ \bigcup_{i=1}^{8} C_{3} & ; S=\{(i j k),(l m n)\}, \\ \bigcup_{i=1}^{6} C_{4} & \{S=\{(i j)(k l),(m n)(p q)\} \\ & \text { and }\{(i j k l),(m n p q)\} .\end{cases}
$$

\section{Proof}

The proof is similar to the proof in the previous proposition.

\section{Theorem 1}

Let $S_{4}$ be the symmetric group of order 24 and $S^{(1)}$ be the subset of $S_{4}$ of valency one. Then, the energy of the Cayley graph of $S_{4}$ with respect to the subset $S^{(1)}$, denoted as $E\left(\operatorname{Cay}\left(S_{4}, S^{(1)}\right)\right)$ is 24 .

\section{Proof}

Consider the symmetric group of order 24, $S_{4}$. By Proposition 3, the Cayley graph, $\operatorname{Cay}\left(S_{4}, S^{(1)}\right)=\bigcup_{i=1}^{12} K_{2}$. By Proposition 1, the adjacency spectrum of a complete graph, $K_{n}$ is $\left\{(n-1)^{1},(-1)^{n-1}\right\}$. Thus, the adjacency spectrum of $\mathrm{U}_{i=1}^{12} K_{2}$ is $12\left\{(2-1)^{1},(-1)^{2-1}\right\}$. This gives the eigenvalues, $\lambda_{i}= \pm 1$ with multiplicity 12 . Therefore, $E\left(\operatorname{Cay}\left(S 4, S^{(1)}\right)\right)=$ $12(1)+12(|-1|)=24$.

\section{Theorem 2}

Let $S_{4}$ be the symmetric group of order 24 and $S^{(2)}$ be the subset of $S_{4}$ of valency two. Then, the energy of the Cayley graph of $S_{4}$ with respect to the subset $S^{(2)}$, denoted as $E\left(\operatorname{Cay}\left(S_{4}, S^{(2)}\right)\right)$ is given in the following:

$$
\left\{\begin{array}{c}
32 ; S=\{(i j),(k l)\} \text { and }\{(i j k),(l m n)\}, \\
12+12 \sqrt{2} ; S=\{(i j),(k l)(m n)\}, \\
24 \quad ; S=\{(i j),(i j)(k l)\},\{(i j)(k l),(m n)(p q)\} \\
\text { and }\{(i j k l),(m n p q)\} .
\end{array}\right.
$$

\section{Proof}

Consider the symmetric group of order 24, $S_{4}$. By Proposition $4, \operatorname{Cay}\left(S_{4}, S^{(2)}\right)$ is given in the following:

$$
\operatorname{Cay}\left(S_{4}, S^{(2)}\right)= \begin{cases}\bigcup_{i=1}^{4} C_{6} & ; S=\{(i j),(k l)\}, \\ \bigcup_{i=1}^{3} C_{8} & ; S=\{(i j),(k l)(m n)\}, \\ \bigcup_{i=1}^{8} C_{3} & ; S=\{(i j k),(l m n)\}, \\ 6 & ; S=\{(i j),(i j)(k l)\}, \\ \bigcup_{i=1} C_{4} & \{(i j)(k l),(m n)(p q)\} \\ & \text { and }\{(i j k l),(m n p q)\} .\end{cases}
$$

From Proposition 2, the adjacency spectrum of a cycle graph $C_{n}$ is $\{2 \cos (2 \pi j / n) ; j=\{0,1, \ldots, n-1\}$. Thus, the adjacency spectrum of $\operatorname{Cay}\left(S_{4}, S^{(2)}\right)$ is given in the following:

$$
\operatorname{Spec}\left(\operatorname{Cay}\left(S_{4}, S^{(2)}\right)\right)= \begin{cases}4\left\{2 \cos \left(\frac{2 \pi j}{6}\right)\right\} & ; \bigcup_{i=1}^{4} C_{6}, \\ 3\left\{2 \cos \left(\frac{2 \pi j}{8}\right)\right\} & ; \bigcup_{i=1}^{3} C_{8}, \\ 8\left\{2 \cos \left(\frac{2 \pi j}{3}\right)\right\} & ; \bigcup_{i=1}^{8} C_{3}, \\ 6\left\{2 \cos \left(\frac{2 \pi j}{4}\right)\right\} & ; \bigcup_{i=1}^{6} C_{4} .\end{cases}
$$

Therefore, the energy of the Cayley graphs, $E\left(\operatorname{Cay}\left(S_{4}, S^{(2)}\right)\right)$ is given in the following: 


$$
\left\{\begin{array}{c}
32 ; S=\{(i j),(k l)\} \text { and }\{(i j k),(l m n)\}, \\
12+12 \sqrt{2} ; S=\{(i j),(k l)(m n)\}, \\
24 \quad ; S=\{(i j),(i j)(k l)\},\{(i j)(k l),(m n)(p q)\} \\
\text { and }\{(i j k l),(m n p q)\} .
\end{array}\right.
$$

\section{Example}

Let $S_{4}$ be the symmetric group of order 24 and $S^{(1)}=\{(13)\}$ be the subset of $S_{4}$. Then, the Cayley graph of $S_{4}$ with respect to the subset $S^{(1)}$, denoted as $\operatorname{Cay}\left(S_{4},\{(13)\}\right)$ is $\bigcup_{i=1}^{12} K_{2}$. Therefore, the energy of the Cayley graph, $E\left(\operatorname{Cay}\left(S_{4},\{(13)\}\right)\right)$ is 24 .

\section{Proof}

Consider the symmetric group of order $24, S_{4}$ and $S^{(1)}=$ $\{(13)\}$ be the subset of $S_{4}$. Then, by Definition 2, the vertex $x$ is connected to $s x$ where $s \in S$.

$$
\begin{aligned}
& (1)-(13) \text { since }(13)(1)=(13) \\
& (12)-(123) \text { since }(13)(12)=(123) \\
& (14)-(143) \text { since }(13)(14)=(143) \\
& (23)-(132) \text { since }(13)(23)=(132) \\
& (24)-(13)(24) \text { since }(13)(24)=(13)(24) \\
& (34)-(134) \text { since }(13)(34)=(134) \\
& (124)-(1243) \text { since }(13)(124)=(1243) \\
& (142)-(1423) \text { since }(13)(142)=(1423) \\
& (1234)-(12)(34) \text { since }(13)(1234)=(12)(34) \\
& (1324)-(243) \text { since }(13)(1324)=(243) \\
& (1342)-(234) \text { since }(13)(1342)=(234) \\
& (1432)-(14)(23) \text { since }(13)(1432)=(14)(23) .
\end{aligned}
$$

The connected elements form the $\operatorname{Cay}\left(S_{4},\{(13)\}\right)$ is $\mathrm{U}_{i=1}^{12} K_{2}$ as illustrated in the following figure.

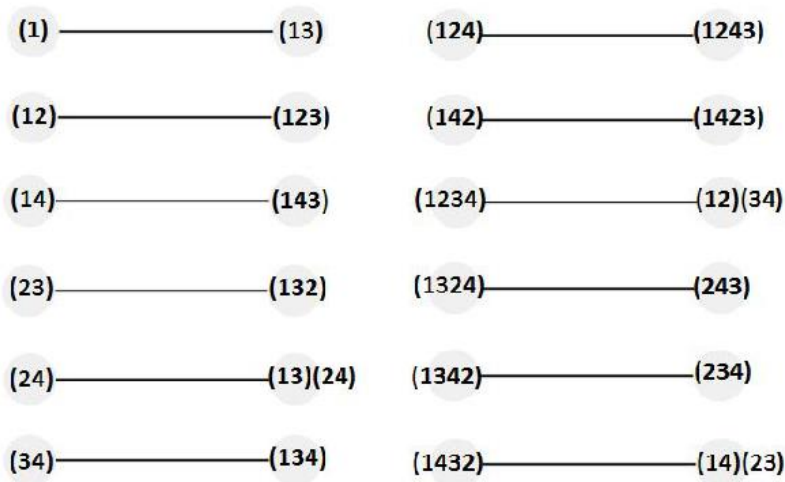

Figure 1. $\operatorname{Cay}\left(S_{4},\{(13)\}\right)$

By the definition of the adjacency matrix, $A\left(\operatorname{Cay}\left(S_{4},\{(13)\}\right)\right)=$

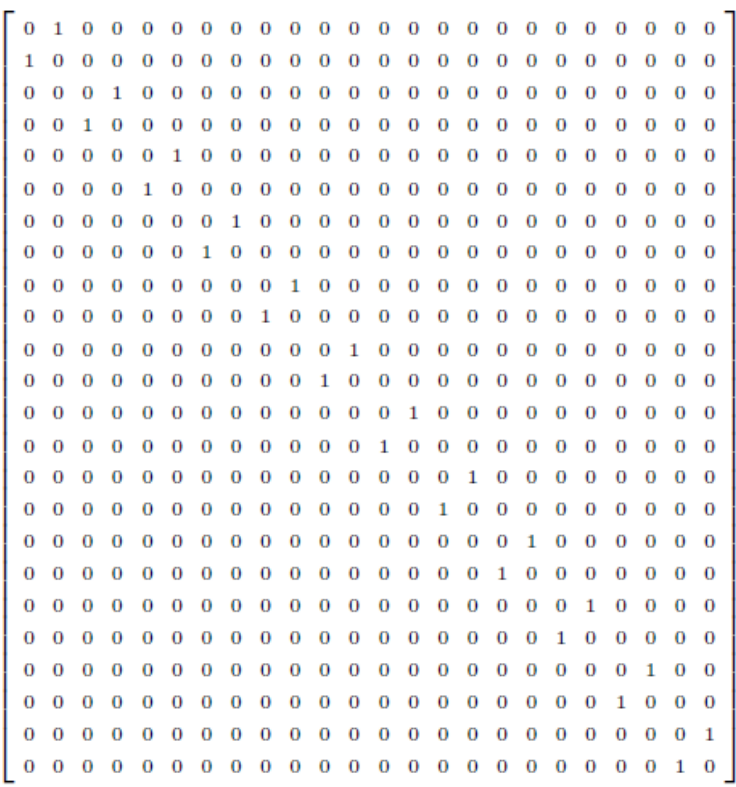

From the adjacency matrix, the characteristic polynomial is found to be $f(\lambda)=\lambda^{24}-12 \lambda^{22}+66 \lambda^{20}-220 \lambda^{18}+495 \lambda^{16}-$ $792 \lambda^{14}+924 \lambda^{12}-792 \lambda^{10}+495 \lambda^{8}-220 \lambda^{6}+66 \lambda^{4}-12 \lambda^{2}+$ 1.

By using the generalization of spectrum of a complete graph, the eigenvalues can be found as \pm 1 with multiplicity 12. Therefore, the energy of the Cayley graph, $E\left(\operatorname{Cay}\left(S_{4},\{(13)\}\right)\right)=12(1)+12(|-1|)=24$.

\section{SUMMARY}

As a conclusion, the energy of the Cayley graphs for the symmetric group of order 24 with respect to the subsets $S$ of valency one and two have been found and are summarized as in the following table.

Table 1. Energy of Cayley graphs of $S_{4}$

\begin{tabular}{|c|c|c|c|}
\hline$|S|$ & Subset $S$ & $\operatorname{Cay}\left(S_{4}, S\right)$ & Energy \\
\hline \multirow{1}{*}{1} & $\{(i j)\},\{(i j)(k l)\}$ & $\bigcup_{i=1}^{12} K_{2}$ & 24 \\
\cline { 2 - 4 } & $\{(i j),(k l)\}$ & $\bigcup_{i=1}^{4} C_{6}$ & 32 \\
\cline { 2 - 4 } & $\{(i j),(i j)(k l)\}$ & $\bigcup_{i=1}^{6} C_{4}$ & 24 \\
\cline { 2 - 4 } & $\{(i j),(k l)(m n)\}$ & $\bigcup_{i=1}^{3} C_{8}$ & 12 \\
\cline { 2 - 4 } & $\{(i j)(k l),(m n)(p q)\}$ & $\bigcup_{i=1}^{6} C_{4}$ & 24 \\
\hline
\end{tabular}




\begin{tabular}{|c|c|c|c|}
\hline \multirow{2}{*}{$(i j k),(l m n)$} & $\bigcup_{i=1}^{8} C_{3}$ & 32 \\
\hline & $\{(i j k l),(m n p q)\}$ & $\bigcup_{i=1}^{6} C_{4}$ & 24 \\
\hline
\end{tabular}

\section{ACKNOWLEDEGMENTS}

The authors would like to express their appreciation to Malaysian Mathematical Science Society (PERSAMA) for the support of sponsor and Research Management Centre (RMC), Universiti Teknologi Malaysia (UTM) Johor Bahru for the financial funding through the Research University Grant (GUP) Vote No. 13J82. The first author would also like to express her appreciation to UTM for the financial support under Zamalah Scholarship. 


\section{REFERENCES}

Adiga, C., \& Ariamanesh, H. 2012, Some properties of Cayley graphs on symmetric groups $S_{n}$. International Journal of Algebra, 6(17), 807-813.

Babai, L., \& Seress, A. 1988, On the diameter of Cayley graphs of the symmetric group. Journal of combinatorial theory, Series A, 49(1), 175-179.

Bapat, R. B. 2010, Graphs and matrices. New York (NY): Springer.

Beineke, L.W., \& Wilson, R. J. 2004, Topics in algebraic graph theory. Vol. 102. USA: Cambridge University Press.

Brouwer, A. E., \& Haemers, W. H. 2011, Spectra of graphs. Springer Science and Business Media.

Daianu, M., Mezher, A., Jahanshad, N., Hibar, D. P., Nir, T. M., Jack, C. R., Weiner, M. W., Bernstein, M. A., \& Thompson, P. M. 2015, Spectral graph theory and graph energy metrics show evidence for the Alzheimers disease disconnection syndrome in APOE-4 risk gene carriers. Proc. IEEE Int. Symp. Biomed Imaging 2015, 458461.

Dehmer, M. Li, X., \& Shi, Y. 2015, Connections between generalized graph entropies and graph energy. Complexity, 21, 3541.

Friedman, J. 2000, On Cayley graphs on the symmetric group generated by tranpositions. Combinatorica, 20(4), 505-519.
Ghorbani, M., \& Nowroozi-Larki, F. 2018, On the Spectrum of Cayley Graphs Related to the Finite Groups. Filomat, $31(20)$.

Gutman, I. 1978, The Energy of Graph. Der. Math. Stat. Sekt. Forschungszent Graz., 103, 1-22.

Konstantinova, E. 2008, Some problems on Cayley graphs. Linear Algebra and its Applications, 429(11), 2754-2769.

Lakshmivarahan, S., Jwo, J. S., \& Dhall, S. K. 1993, Symmetry in interconnection networks based on Cayley graphs of permutation groups: A survey. Parallel Computing, 19(4), 361407.

Pemmaraju, S., \& Skiena, S. 2003, Computational Discrete Mathematics: Combinatorics and Graph Theory with Mathematica. Cambridge University Press.

Robinson, D. J. 1996, A course in the theory of groups, 2nd. ed. USA: Springer-Verlag New York, Inc.

Wu, H., Zhang, Y., Chen, W., \& Mu, Z. 2015, Comparative analysis of protein primary sequences with graph energy. Physica A: Statistical Mechanics and its Applications, 437, 249262. 